\title{
Theory Refinement and Natural Language Learning
}

\author{
Hervé Déjean* \\ Seminar für Sprachwissenschaft \\ Universität Tübingen \\ dejean@sfs.nphil.uni-tuebingen.de
}

\begin{abstract}
This paper presents a learning system for iclentifying syntactic structures. This systen relies on the use of background knowledge and default values in order to build up an initial grammar and the use of thoory refinement in order to improve this grammar. This combination provicles a good machine loarning framework for Natural Language Learning. Wo illustrate this point with the presentation of ALLiS, a learning system which gencrates a regular expression grammar of non-recursive phrases from bracketed corpora.
\end{abstract}

\section{Introduction}

Applying Machine I Jaming techniques to Natumal Language Processing is a booming domain of rescarch. One of the reasons is the development of corpora with morpho-syntactic and syntactic annotation (Marcus et al., 1993), (Sampson, 1995). One recont popular subtask is the learning of non-recursivo Nouns Phrases (NP) (Ramshaw and Marcus, 1995), (Tjong Kim Sang and Vecustra, 1999), (Muño\% ot al., 1999), (Group), 1998), (Cardic and Picrec, 1999), (Buchhol $\%$ et al., 1999).

When other learning techniques (symbolic or statistical) are widely used in Natural Language Loarning, theory refincment (Abocker and Schnid, 1996), (Mooney, 1993) soems to be ignored (cxcept (Brunk and Pazzani, 1995)). Theory refinement consists of improving an existing knowledge base so that it better accords with data. No work using theory refinement applied to the grammar lcarning paradigm seems to have beon developed. We would like to point out in this article the adequacy between theory refinement and Natural Language Learning.

To illustrate this claim, we present ALLiS (Architecture for Learning Linguistic Structures), a learning system which uses theory refinement in order to learn non-recursive noun phrases (also callod base noun phrases) and non-recursive verbal phrases. We will show that this technique combined with the

\footnotetext{
* This research is funcled be the l'MR network lecurning Computational Grammats ww. $\operatorname{lcg}$-ww. uia.ac.be/lcg/
}

use of default values provides a good architecture to learn natural language structures.

This article is organised as follows: Section 2 gives an overview of theory refincment. Section 3 explains the advantage of combining default values and theory refincment to build a learning system. Section 4 describes the general characteristics of ALLiS, and Section 5 explains the learning algorithm. 'The evaluation of ALLiS is clescribed Section 6. The examples which illustrate this article correspond to English NI's.

\section{Theory Refinement}

We present here a briof introduction to theory refincment. For a more detailed presentation, we refor the reader to (Abocker and Schnid, 1996), (I3runk, 1996) or (Ourston and Mooncy, 1990). (Mooncy, 1993) defines it, as:

Theory refinoment systems developed in Machine Joarning antomatically modify a Knowledge 13ase to render it consistent with a set of classified training examples.

This tochnique thus consists of improving a given Knowledge Base (here a grammar) on the basis of examples (here a trechank). Some impose to modify the initial knowledge base as little as possible. Applied in conjunction with existing learning technicues (Explanation-Based Learning, Incluctive Logic P'rogramming), TR seems to achieve better results than these techniques used alone (Mooncy, 1997). Theory refincment is mainly used (and has its origin) in Knowledge Based Systems (KBS) (Craw and Sleeman, 1990). It consists of two main steps:

1. Build a more or less correct grammar on the basis of background knowledge.

2. Refine this grammar using training examples:
(a) Identify the revision points
(b) Correct them

The first step) consists of acquiring an initial grammar (or more gencrally a knowledge base). In this work, the initial grammar is automatically induced 
from a tagged and bracketed corpus. The second step (the refinement) compares the prediction of the initial grammar with the training corpus in order to firstly identify the revision points, i.e. points that are not correctly described by the grammar, and secondly, to correct these revision points. The orror identification and refinement operations are explained Section 5.3.

The main difference between a TR system and other symbolic learning systems is that a TR system must be able to revise existing rules given to the system as background knowledge. (A system such as TBL (Brill, 1993) can not be considered as TR since it only acquires new rules). In the case of other techniques, new rules are lcarned in order to improve the general efficiency of the system (sclection of the "best rule" according to a preference function) and not in order to correct a specific rule.

\section{Theory Refinement, Default values and Natural Language Learning}

This section explains how default values combined with theory refinement can provide a good machine learning framowork for NLP.

\subsection{The Use of Default Values}

The use of default values is not new in NLP (Brill, 1993), (Vergne and Giguet, 1998). We can observe that often (but not necessarily) in a language, an element belongs to a predominant class (Vorgne and Giguet, 1998). Some systems such as stochastic models use this property implicitly. Some others use it explicitly. For instance, the general principle of the Transformation-Based Learning (Brill, 1993) is to assign to each clement its most frequent category, and then to learn transformation rules which correct its initial categorisation. A second example is: the parser described in (Vergne and Giguet, 1998). They first assign to each grammatical word a default category (default tag), and then might modify it thanks to local contexts and grammatical rolation assignment (in order to deal with constraints duc to long distance relations which can not be expressed by local contexts).

The main work is done by the lexicon and by default values (even if further opcrations arc obviously necessary)

These approaches are thus different for the disambiguation often used in tagging. The default rules are not numerous (one per tag), easy to automatically generate but they nevertheless produce a satisfactory starting level.

\subsection{The Combination of Default Values with TR}

The idea on which ALLiS relies is the following: a first: "naive grammar" is built up using default values, and then TR is used in order to provide a "more realistic grammar". This initial grammar assigns to each element its default catcgory (the algorithm is explained in Section 5.2). The rules learned are categorisation rules: assign a category to an element (a tag or a word). Since an cloment is automatically assigned to its default category, the system has not to learn the categorisation rules for its category, and just learns categorisation rules which correspond to cases in which the element does not belong to its default category. This minimised the number of rules that have to be learned. Suppose the clement e can belong to several categorios (a frequent case). The first rule learned is the "default" rule: $\operatorname{assign}(e, d c)$, where $d c$ is the default category of $e$. Then ALLiS just learns rules for cases where $e$ does no belong to its default category. The numerous rules concerning the default category are replaced by the simple default rule.

\section{ALLiS}

The goal of ALLiS ${ }^{1}$ is to automatically build a regular expression grammar from a bracketed and tagged corpus $^{2}$. In this training data, only the structures we want, to learn are marked at thcir boundaries by square brackets ${ }^{3}$. The following sentence shows an example of the training corpus for the NP structure (only base-NPs occur inside brackets).

$$
\begin{aligned}
& \text { In/IN [ carly/JJ trading/NN] in/IN [ } \\
& \text { Hong/NNP Kong/NNP ] [ Monday/NNP } \\
& \text { ] } /,[\text { gold/NN ] was/VBD quoted/VBN } \\
& \text { at/IN [ } \$ / \$ 366.50 / \mathrm{CD}] \text { [ an/DT } \\
& \text { ounce/NN ] } / .
\end{aligned}
$$

ALLiS uses an internal formalism in order to represent the grammar rules. In order to parse a toxt, a module converts its formalism into a regular expression grammar which can be used by a parser using such representation (two modules exist: one for: the CASS parsor (Abnoy, 1996) and one for XIST (Karttunen et, al., 1997)).

Following the principle of theory refinement, the learning task is composed of two steps. The first step is the generation of the initial grammar. This grammar is generated from examples and background knowledge (Section 5). This initial grammar provides an incomplete and/or incorrect analysis of the data. The second step is the refincment of this grammar (Section 5.3). During this step, the validity of the grammar rules is checked and the rules are improved (refined) if necessary. This improvement corresponds to find contexts in which elements which

\footnotetext{
${ }^{1}$ http://www .sfb441.uni-tuebingen.de/ dejean/ chunker.html.

${ }^{2}$ The WSJ corpus (Marcus et al., 1993).

${ }^{3}$ (Muñoz et al., 1999) showed that this representation tends to provide better results than the representation used in (Ramslaw and Marcus, 1995) where each word is tagged with a tag $\mathrm{I}$ (inside), $\mathrm{O}$ (outside), or $\mathrm{B}$ (breaker).
} 
are considered to be members of the structure do not bolong to this structure (and reciprocally).

We give here a simple example to illustrate the learning process. The first step (initial grammar gencration) categorises the tag $J J$ (arljective) as belonging by default to the NP structure if it occurs before a noun. The second step) (refinement) finds out that some adjectives clo not obey to these rules ${ }^{4}$. The refinement is triggered in order to modify the default rule so that these exceptions can be correctly processed.

Thus, the learning algorithm simply consists of categorising the elements of the corpus (tags and words) into specific categorics, and this categorisation allows the extraction of the structures we want, to learn. These ategories are explained in the next section.

\section{The Learning System}

\subsection{The Background Knowledge}

In order to caso the learning, the system uses background knowledge. 'This knowledge provides a for-mal and general description of the structures that ALLiS can learn. Wo suppose that the structures are composed of a nucleus with optional left and right adjuncts. We here give informal definitions, the formal/distributional ones are given in Section 5.2.

The nucleus is the head of the structure. We authorise the presence of several nuclei in the same structiure.

All the other elements in the structiure (excepti the linker) are considered as adjuncts. They are in dependence relation with the head of the structure. The adjuncts are characterised by their position (left/right) relative to the nucleus.

A linker is a special olement, which builds an endocentric structure with two elements. It usually corresponds to coorlination ${ }^{5}$.

An element (nuclens or adjunct) might possess the break property. This notion is introduced (as in (Ramshaw and Marcus, 1995), (Muñoz et al., 1999)) in order to deal with sequences where adjacent nuclei compose several structures (Section 5.2.4).

This pattern can be seen as a variant of the $\mathrm{X}$ bar template (Head, Spec and Comp), which was alroady used in a loarning systom (Bcrwick, 1985) (although Comp is not useful for the non-reculsive structures).

The possible different categories of an element are summarised in ligure 5.1 .

The following sentence shows an example of categorisation (elements which do not appear in the structure (NP) arc tagged $(\mathrm{O})$ :

\footnotetext{
${ }^{4}$ lor example: [ the/J'T 7/C,I) \%/NN benchmark/NN issuc/NN] due/JJ [ October/NNI' 1999/CD].

"Only linkers occurring between the two coordinated elements are processed.
}

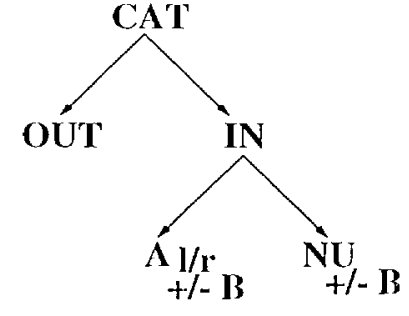

Figure 1: The different categories.

Ino carly $\mathrm{A}_{\mathrm{B}+}$ trading $\mathrm{NU}_{\mathrm{O}}$ inong $\mathrm{H}_{\mathrm{NU}}$ Kong $\mathrm{NU}_{\mathrm{N}}$ MonclaynU,+ 0 gold $\mathrm{Nu}_{\mathrm{Nu}}$ was 0 quotedo at $0 \$_{\mathrm{A}}$ $366.500_{\mathrm{NU}} \mathrm{in}_{\mathrm{AB}+}$ ounce $\mathrm{NU}^{-0}$

The structure is formally defined as:

$$
\begin{aligned}
& \mathrm{S} \rightarrow \mathrm{A}_{1, \mathrm{~b}+} *\left[\mathrm{~A}_{1, \mathrm{~b}-} * \mathrm{NU}_{\mathrm{b},-} \mathrm{A}_{\mathrm{r}, \mathrm{b}-}{ }^{*}\right]+\mathrm{A}_{\mathrm{r}, \mathrm{b}+} * \\
& \mid A_{1, b+} * A_{1, b-} * N U_{b-+} A_{r, b-} * A_{1, b+} *
\end{aligned}
$$

The symbol * corresponds to the Kleene star. For the sake of legibility, wo do not, introduce linkers in this expression. But ach symbol $X$ (NU, $A)$ cam be defined by the rule ${ }^{\prime} \rightarrow X \mid X l X$ where $l$ is the list of linkers. The symbols $B+$ and $B$ - indicate whether the element has the breaker property or not.

Since the corpus does not contiain information about these distributional categories, AluLis has to figure them out. This aategorisation relies on the distributional bohaviour of the elements, and can bo antomatically achioved.

\subsection{The Initial Categorisation}

The general idea to categorise olements is to nese spe(ific contexts which point ont some of the distributional propertice of the category. 'The catcgorisation is a secpuential process. First the nuclei have to be found ont. For each tag of the corpus, we apply the function $f_{\text {nu }}$ (described below). This function selects a list of eloments which are categorised as nuclei. The function $f_{b}$ is applied to this list in order to figure out nuclei which are breakers. Then the adjuncts are found out, and the finction $f_{\mathrm{b}}$ is also applied to them to figure out breakers.

\subsubsection{Categorisation of Nuclei}

The context used to find out the nuclei relies on this simple following observation: A structure requires at least one nucleus? ${ }^{7}$. Thus, the elements that occur alone in a structure are assimilated to nucleus, since a structure requires a nucleus. For example, the tags PRP (pronouns) and NNP (proper nouns) may compose alone a structure (respoctively $99 \%$

\footnotetext{
GrThe regular expression formalism does not allow such rule, and then, this recursion is simulated.

${ }^{7}$ lhe partial structures (structures without nucleus) represent $2 \%$ of all the structures in the training corpus, and then introduce little noise.
} 
and $48 \%$ of these tags appear alone in NP) but the tag J.J appears alone only $0.009 \%$. We deduce that PRP and NNP belong to the nuclei and not .JJ. But this critcrion does not allow the identification of all the nuclei. Some often appear with adjuncts (an English noun (NN) often ${ }^{8}$ occurs with a determiner or an adjective and thus appears alone only $13 \%$ ). The single use of this characteristic provides a continuum of values where the automatic set up of a threshold between adjuncts and nuclei is problematic and depends on the structure. To solve this problem, the idea is to decompose the categorisation of nuclei in two steps. First we identify characteristic adjuncts. The adjuncts can not appear alone since they depend on a nucleus. The function $f_{\text {char }}$ is built so that it provides a very small value for adjuncts. If the value of an element is lower than a given threshold $\left(\theta_{\text {char }}=0.05\right)$, then it is catcgorised as a characteristic adjunct.

$$
f_{\text {char }}(X)=\frac{\sum_{C}[X]}{\sum_{C} X}
$$

$\sum_{C} P$ corresponds to the number of occurrences of the pattern $P$ in the corpus $C$. For example the number of occurrences in the training corpus of the pattern $[J J]$ is 99 , and the number of occurrences of the pattern $J J$ (including the pattern [ JJ]) is 11097. So $f_{\text {char }}(J J)=0.009$, value being low enough to consider JJ as a characteristic adjunct. The list provided by $f_{\text {char }}$ for English NP is:

$$
\left.A_{\text {char }}=\left\{D T, P R P \$, P O S, J . J, J . J R, J J S,{ }^{\prime}, '\right]\right\}
$$

These clemonts can correspond to left or right adjuncts. All the adjuncts are not identified, but this list allows the identification of the nuclei as explained in the next paragraph.

The second step consists of introducing these clements into a new pattern used by the function $f_{\text {nu }}$. This pattern matches elements surrounded by these characteristic adjuncts. It thus matches nuclei which often appear with adjuncts. Since a sequence of adjuncts (as an adjunct alone) can not alone compose a complete structure, $X$ only matches elemonts which correspond to a nucleus.

$$
f_{\mathrm{nu}}(X)=\frac{\sum_{C}\left[A_{\left.\operatorname{char}^{*} X A_{\text {char }^{*}}{ }^{*}\right]}\right.}{\sum_{C} X}
$$

The function $f_{\text {nu }}$ is a good discrimination function between nuclei and adjuncts and provides very low values for adjuncts and very high values for nuclei (table 1).

\subsubsection{Categorisation of Adjuncts}

Once the nuclei are identificd, we can casily find out the adjuncts. They correspond to all the other elements which appear in the context: There are two

\footnotetext{
${ }^{8} \wedge t$ least, in the training corpus.
}

\begin{tabular}{lccc}
\hline $\mathrm{x}$ & Freq $(\mathrm{x})$ & $f_{\text {nu }}(x)$ & nucleus \\
\hline POS & 1759 & 0.00 & no \\
PRP\$ & 1876 & 0.01 & no \\
JJ & 11097 & 0.02 & no \\
DT & 18097 & 0.03 & no \\
RB & 919 & 0.06 & no \\
\hline NNP & 11046 & 0.74 & yes \\
NN & 21240 & 0.87 & yes \\
NNPS & 164 & 0.93 & yes \\
NNS & 7774 & 0.95 & yes \\
WP & 527 & 0.97 & yes \\
PRP & 3800 & 0.99 & yes \\
\hline
\end{tabular}

Table 1: Detection of some nuclei of the English NP (nouns and pronouns).

kinds of adjuncts: the left and the right adjuncts. The contexts used are:

$$
\begin{array}{ll}
{[-\mathrm{NU}]} & \text { for the left adjuncts } \\
{\left[\mathrm{A}_{\mathrm{l}}^{*} \mathrm{NU} \mathrm{U}_{-}\right]} & \text {for the right adjuncts }
\end{array}
$$

If an elcment appears at the position of the underscore, it is categorised as adjunct. Once the left adjuncts are found out, they can be used for the categorisation of the right adjuncts. They thus appear in the context as optional elements (this is helpful to capture circumpositions).

Since the Adjective Phrase occurring inside an NP is not marked in the Upenn treobank, we introduce the class of adjunct of adjunct. The contexts used to find out the adjuncts of the left adjunct are:

$$
\begin{aligned}
& {\left[-A_{l} N U\right] \text { for the left adjuncts of } A_{l}} \\
& {\left[a_{l}^{*} A_{l}-N U\right] \text { for the right adjuncts of } A_{l}}
\end{aligned}
$$

The contexts are similar for adjuncts of right adjuncts.

\subsubsection{The Linkers}

By definition, a linker connects two elements, and appears between them. The contexts used to find linkers are:

$$
\begin{array}{lc}
{[N U-N U]} & \text { linker of nuclei } \\
{[A-A N U]} & \text { linker of left adjuncts } \\
{[N U A-A]} & \text { linker of right adjuncts }
\end{array}
$$

Elements which occur in these contexts but which have already been categorised as nucleus or adjuncts are deleted from the list.

\subsubsection{The Break Property}

A sequence of several nuclei (and the adjuncts which depend on them) can belong to a unique structure or compose several adjacent structures. An element is a breaker if its presence introduces a break into a sequence of adjacent nuclei. For example, the presence of the tag $D T$ in the sequence NN DT JJ NN introduces a break before the tag DT, although the 
sequence NN JJ NN (without I'T) can compose a single structure in the training corpus.

$$
\begin{aligned}
& \ldots[\text { the/D' coming/VBG week/NN] } \\
& \text { [thc/D'T foreign/J.J exchange/NN mar- } \\
& \text { ket/NN] ... }
\end{aligned}
$$

The tag $D T$ introduces a break on its left, but some tags can introduce a break on their right or on their left and right. For instance, the tag WDT (NU by default) introduces a broak on its left and on its right. In other words, this tag can not belong to the same structure as the precoding adjacent nucleus and to the same structure as the following adjacent nucleus.

$$
\begin{aligned}
& \ldots \text {. [ railroads/NNS and/CC trucking/NN } \\
& \text { companies/NNS ] [ that/WDT ] bo } \\
& \text { gan/VIBD in/IN [ 1980/CD ] . . } \\
& \ldots \text { in/JN [ which/WDT ] [ peo]le/NNS ] } \\
& \text { gencrally/RI3 aro/VBP ... }
\end{aligned}
$$

In order to detect; which tag has the break property, we build up two functions $f_{b}$ left and $f_{b}$ right.

$$
\begin{aligned}
& f_{\mathrm{b} \operatorname{lefl}}(X)=\frac{\left.\sum_{c} N U\right][X}{\sum_{c_{w b}} N U X} \\
& f_{b \text { right }}(X)=\sum_{\left.\sum_{\text {wb }} X\right][N U} \frac{X]}{N U} \quad N U:\{\text { nuclei }\} \\
& C_{\text {wb }}: \text { corpus without brackets }
\end{aligned}
$$

Theso functions are used to compute the break property for mucloi, but also for adjuncts (In this case, the pattern $\mathrm{X}$ is completed by adcling the alement NU to the lof or to the right of $\mathrm{X}$ (the potential adjunct) according to the kind of adjunet (left or: right adjunct)). The table 2 shows some values for some tags. An olement can be a left breaker (D'), a right breaker (no example for English NP at the tag level), or both (PRP). The break property is generally woll-marked and the throshold is easy to set up (0.66 in practico).

\begin{tabular}{lcc}
\hline TAG & $f_{\mathrm{b} \text { loft }}$ & $\mathrm{f}_{\mathrm{b}}$ right \\
\hline JT. & 0.97 (yes) & $0.00(\mathrm{no})$ \\
PRP & 0.97 (yes) & $0.68($ yes $)$ \\
POS & 0.95 (yes) & $0.00($ no) \\
PRTP & 0.94 (yes) & $0.00($ no) \\
J.J & 0.44 (no) & $0.00($ no) \\
NN & 0.04 (no) & $0.11($ no) \\
NNS & 0.03 (no) & $0.14($ no) \\
\hline
\end{tabular}

Table 2: Brealker determination. Values of the functions $f_{b \text { left }}$ and $f_{\text {b right }}$ for some olements.

In the refincment step) (Section 5.3), the breaker property can be extended to words. Thus, the word yesterday is considered as a right breaker, although its tag (NN) is not.

\subsection{The Refinement Step}

\subsubsection{The notion of reliability}

The preceding functions identify the category of an clement when it occurs in the structure. But an clement can occur in the structure as well as out of the structure. For example, the tag VBG is only considcred as adjunct when it occurs in the structure. Nevertheless, it mainly occurs out of the structure $(84 \%$ of its occurrences). If an element mainly ${ }^{9}$ occurs out of the structure, it is considered as non-reliable and its default catcgory is OUT'. For each element occurring inside the structure, its reliable is tested. The initial grammar corresponds to the grammar which only contains the reliable elements. Its precision and its recall are around $86 \%$.

How is determined the reliability of an element? This notion of reliable element is contextual and depends on the category of the element.

For the nuclei, the context is empty. We just compute the ratio between the number of occurrences in the structure over the number of occurrences occurring ottiside of the structure.

For the adjuncts, the context includes an adjacent nucleus (on the right for left adjuncts or on the left for right adjuncts). For instance, the tag .J.J is categorised as left adjunct for the English NP. It appears 9617 times before a nucleus and 9489 times in the structure. It is thus considered as reliable, and its default category is left, adjunct. In the case where the tag J.J ocenus without nucleus on its right: (a predicative uso), it is not considered as adjunet: and this kind of occurrences is not used to determine the reliability of the element. On the contary, the tag VI3G appears 468 times before a nucleus, but, in this context, it occurs only 138 times in the structure. This is not cnough (29\%) to consider the element as a reliable loft adjunct, and thus its default category is OUT. For the adjunct of adjunct, the context includes adjunct and nucleus.

\subsubsection{Detection of errors}

Once the initial grammar is built up, its emors have to be corrected. 'The detection of the errors corresponds to a miscategorisation of a tag. An automatic cror done by the initial grammar is to wrongly analyse the structures composed with non-reliable elements (false negative oxamples). Each time that a non-rcliable element occurs in tho structure corresponds to an error. For instance, the initial grammar can not comectly recognise the following sequence as an NP, the default category of the tag $V B G$ being OUT (outside the structure):

$$
\text { ... [the/1) } \Gamma \text { coming/VBG weck/NN] ... }
$$

\footnotetext{
"rhe threshold used is of $50 \%$.
} 
The second kind of crrors corresponds to sequences wrongly recognised as structures (false positive examples). This kind of error is generaled by reliable elements which exceptionally do not occur in the structure. In the following example, order $/ N N$ occurs outside of the structure, although the default category of the tag $N N$ is NU (muclens), and thus the initial grammar recognises an NP.

$$
\ldots \text { in/IN order/NN to } / \mathrm{TO} \text { pay/VB ... }
$$

\subsubsection{Correction}

In both kinds of errors, the same technique is used to correct them. Hor this purpose, ALLiS disposes of two operators, the contextualisation and the lexicalisation.

The contextualisation consists of finding out contexts in order to fix the errors. The iden is to add constraints for recategorising non-roliable elements as reliable ${ }^{10}$. The presence of some specific elements can completely change the behaviour of a lag. The Lable 3 shows the list of contexts where the tag $V B N$ is not categorised as OU'I but as lelt Adjunct.

\begin{tabular}{lll}
\hline PRP\$ & VBG & NU \\
IN [ & VBG & NU \\
DT & VBG & NU \\
JJ & VBG & NU \\
POS & VBG & NU \\
VBG [ & VBG & NU \\
\hline
\end{tabular}

Table 3: Some contexts where the non-reliable clement, VBN becomes reliable.

For cach tag occurring in the structure, all the possible contexts ${ }^{11}$ are generated. For the nonreliable tags (first kind of error), we caluate the reliability of them contextually, and we delete tho contexts in which the tag is still non-roliable (the list of contexts can be empty, and in this case the arror can not be fixed). For the reliable tags (second kind of error), we keep the contexts in which the tag is catcgorised OU'I.

The lexicalisation consists of introducing lexical information: the word level. Some words can have a specific behaviour which does not appear at the Part-Of-Specch (POS) level. For instance, the word yesterday is a left breaker, behaviour which can not be figured ont at the POS level (lable 4). The introduction of the lexicalisation improves the result by $2 \%$ (Section 6 ).

The lexicalisation and the contextualisation can be combined when both separately are not powerful cnough to fix the error. For example, the word about tagged RB (default category of RB: OUT) followed

\footnotetext{
10rlhe same technique is used in (Sima'an, 1997).

${ }^{1}$ ' The contexts depend on the category of the tag, but, are just composed of one oloment.
}

\begin{tabular}{llc}
\hline word(context) & default cat. & new cat. \\
\hline about/RB ( - CD) & OUT & $\mathrm{Al}_{1, \mathrm{~B}+}$ \\
order (IN - TO) & $\mathrm{NU}$ & $\mathrm{OUT}$ \\
yesterday/NN & $\mathrm{VU}_{\mathrm{B}-}$ & $\mathrm{NU}_{\mathrm{B}+}$ \\
operating/NBG & $\mathrm{OUT}$ & $\mathrm{A}_{\mathrm{l}, \mathrm{B}-}$ \\
last/JJ & $\Lambda_{l, B}$ & $\mathrm{Al}, \mathrm{B}+$ \\
\hline
\end{tabular}

Table 4: Somo specific lexical behaviours.

by the tag $C D$ is recategorised as left arljunct and left broakor (Table 4).

\section{Evaluation}

We now show some results and give some comparisons with other works (Table 5). The results are quite similar to other approaches. Two rates are measurcd: precision an recall.

$$
\begin{aligned}
& R=\frac{\text { Number of correct proposed palterns }}{\text { Number of compct patterns }} \\
& P=\frac{\text { Number of corret proposcl patterns }}{\text { Number of proposed pratterns }}
\end{aligned}
$$

The training data are composed of the sections 15-18 of the Wall Strcet Journal Corpus (Marcus ot a]., 1993), and wo use the section 20 for the test corpus ${ }^{12}$. The data is tagged with the Brill tagger. The works generating symbolic rules like ALLiS are (Ramshaw and Marcus, 1995) (TransformationBased learning) and (Cardic and Pierco, 1998) (crror-driven pruning of troobank grammars). ALLiS provides better results than them. (Argamon et al., 1998) use a Memory-Based Shallow Learning; system, (Tjomg Kim Sing and Veenstra, 1999) the Memory-l3ased Learning method and (Muñoz ct al., 1999) uses a network of linear functions. The latter work seems to integrate better lexical information since ALLis gets better results with POS only.

\begin{tabular}{llll}
\hline & & POS only & with words \\
\hline N1 & MPRZ99 & $90.3 / 90.9$ & $92.40 / 93.10$ \\
& ALLiS & $91.0 / 91.2$ & $92.56 / 92.36$ \\
& TV99 & & $92.50 / 92.25$ \\
& ADK98 & $91.6 / 91.6$ & \\
& lMM5 & $90.7 / 90.5$ & $92.3 / 91.8$ \\
& CP98 & $91.1 / 90.7$ & \\
\hline VP & ALLiS & $91.39 / 90.52$ & $92.15 / 91.95$ \\
\hline
\end{tabular}

Table 5: Results for NP and VP structures (precision/rocall).

The main errors dome by ALLis are due to errors of tagging (the corpus is tagged with the Brill tagger) or errors in the bracketing of the training corpus. Then, the second type of errors concerns

\footnotetext{
${ }^{12}$ This data set is available via $\mathrm{ftp} / / \mathrm{ftp}$.cis upenn.edu/ pub/chunker/.
} 
the coordinated structures. 'Ihese two types errors compespond to at\% of the overall mors. Wo cun find the sinne typology in other works (Rannshaw and Marcus, 1995), (Candio and l'ience, 1998). Wo: diel some tries in order to manually improve the final grammax, but the only type of errors which cau be manually improved concerns the problem of the quotation marks (the inprowement is about of $0.2 \%$ in precisions and recall).

\begin{tabular}{|c|c|c|}
\hline Froo types & $\#:$ & 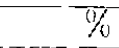 \\
\hline tagging/hracketing errors & 57 & $28.5 \%$ \\
\hline coordination & 45 & $22.5 \%$ \\
\hline gertind & 15 & $7.5 \%$ \\
\hline adverb & 13 & $6.5 \%$ \\
\hline appositives & 13 & $6.5 \%$ \\
\hline quolation marks, punctuations & 10 & $5 \%$ \\
\hline past, participle & 9 & $1.5 \%$ \\
\hline that (IN) & 6 & $3 \%$ \\
\hline
\end{tabular}

Iable 6: 'Typology of the 200 tiwst crrors.

\section{References}

Androns Abocker and Klans Schmicl. 1996. Trom theory refinement lo kb mantenance: a posilion statement. In $E^{\prime} C^{\prime} A I^{\prime} B 6$, Judalpest, Ilungary.

Steven Abney. 1990. l'artial parsing via finite..state cascades. In Procectings of the HSSLIT 96 Rolunst l'arsing Workshop.

Sllomo Arganon, Ido Dagan, anel Yumal lisymolowski. 1998. A momory-based approatch to leaning shallow natural langnage patterns. Th CoLING'98, Montréal.

Robert C. Berwick 1985. The acquistion of syntactic lenouledge. MIT press, C'ambritge.

IEric Brill. 1993. A Corpus-Based Apmrourh to Lanquage Learning. Ph.D. thesis, Depatment of Computer ancl Information Science, University of Pennsylwain.

Clifford Alan I3runk and Mishael Pariani. 1995. A lexically based semantics bias for theory revision. In Morgan Kandlinan, oditor, 'Twelfth Inlernational Conference on Machine Koraming, pages 81-89.

Cliflord Alan Brunk. 1996. An investigation of Knowledge Intensive Appronches to Concepl Learning and Theory Refinement. Pl..D. thesis, University of California, Irvine.

Sabine Buchhol\%, Jorn Vecnstra, and Walter Daclemans. 1999. Cascenled grammatical relation assignment. In l'rocedings of $\mathrm{EMNLP} / V L C-99$, pages pp. 239246 , University of Maryland, USA.

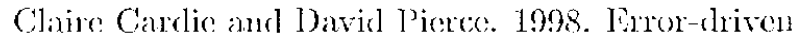
pruning of tredbank grammans for base nom phrase identification. In l'rocecedings of the $17 \mathrm{th}$
Intemalional Conference on Compndational Linguistics (COLIN $(r-A C L, 98)$.

Gaire Cardie and David l'iere. 1999. The role of lexicalization and proming for base nom phrnse

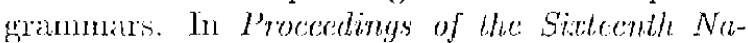
tional Conference on Artificial Intelligence.

Susan (baw and J). Sleonlan. 1990. Antomating tho rofinement of knowledge-based systems. In Proceetings of the tCAI'OO Conference, pages 167 . 172.

The X X'AG Rescarch Group) 1998. A lexicalized treo adjoining, grammar for english. Technical Report MRCS 98-18, University of Ponnsylyania.

Jauri Karttunen, 'Iannis Gal, and André Konpe. 1997. Xerox finite-stante tool. Technical report, Xerox liesearch Gentre Finope, (t)enoble.

Mitchell Mancens, Bóntrice Santorini, and Mare Anu Marcinkiowio. 1993. Building a largo annotated corpus of english: the penn treobank. Computiotional Singuistics, 19(2):313 330.

Raymond J. Mooney. 1993. Induction orer the unexplained: Using overly-general domain theories to and concept learning. Machine Learning, 10:79.

Raynond .J. Mooncy. 1997. Intluctive logice programming for natumal language procossing. In Sixth International Inductive Logir: Programming Workshop, pares 205 221, Stocklohn, Sweden.

Mancia Muño, Vasin l'nuyakanok, Jan Roth, and Dav Zinak. 1999. A loarning approach to shillow parsing. In Proced dings of EMNLP-WVLC'g9.

Dirk (Ourstom and Raymond Mooney: 1990. Changing the rules: $A$ comprehensive apporath to theory refinement. In Procedings of thre light National Conferenee on Artificial Sntelligence, pages 815 820 .

Lance A. Rannshaw and Mitchell P. Mancos. 1995. Text chunking using tarnsformation-based loarning. In ACI, Third Workshop on Very Large Compora, pages 8291 .

Geofliey Sampson. 1995. English for the Compuler. The SUSANNS Corpus and Analylie Scheme. Oxford: Clinenelon Puess.

Khalil Sima'au. 1997. Explanation-based learning of data orionted parsing. In T. Mark Ellison, editor, Computational. Notural Language Learning

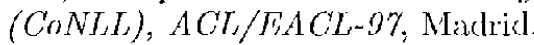

Jirik Tong Kim Sang and Jorn Veonstra. 1999. Representing text chunks. In Proceedings of FACI'D9, Association for Computational Linguistics, Bergen.

Jacques Vergno and Emmanuel Giguel. 1998. Regards theoricuess sur lo "laggging". In proceedings of Truitement Automatique des Langues $\mathrm{No}$ turelles (TALN 1998), I'aris. 